\title{
EDITORIAL
}

\section{A promising era for epigenetic research: revealing the molecular signature of neuropsychiatric disorders}

\author{
Thiago Wendt Viola, ${ }^{1}$ (iD Gabriel Rodrigo Fries ${ }^{2}$ (iD \\ ${ }^{1}$ Developmental Cognitive Neuroscience Lab, Pontifícia Universidade Católica do Rio Grande do Sul (PUCRS), Porto Alegre, RS, Brazil. \\ ${ }^{2}$ Department of Psychiatry and Behavioral Sciences, University of Texas Health Science Center at Houston, Houston, TX, USA.
}

This is an exciting moment for the field of epigenetics. The idea that epigenetic changes could serve as biomarkers of disease detection, prognosis, and treatment response has been recently boosted in many health-related fields, including neuropsychiatry. Epigenetic mechanisms have traditionally been defined as molecular processes associated with cell lineage specification, particularly during early development. In recent decades, this definition has expanded with the recognition that a variety of these processes are also dynamic and can integrate intrinsic and extrinsic signals into the genome, which in turn facilitates an organism's adaptation to its environment through sustained changes in gene expression. Epigenetic mechanisms, including the repertoire of DNA, histone and chromatin modifications, as well as the role of non-coding RNAs and DNA/RNA editing, might be rapid and longlasting, providing fine-tuned regulation of experienceinduced gene expression.

Our understanding of the role of these mechanisms in mental disorders and brain processes has increased considerably over the last two decades. Evidence has begun to emerge from pre-clinical models that exposure to learning events and adverse environments could regulate transcription of well-known genes implicated in neuroplasticity (e.g., BDNF) and stress response (e.g., NR3C1) in the brain. ${ }^{1}$ In the era of genome-wide association, epigenome-wide studies have been revealing new candidate loci involved in the molecular signature of psychiatric disorders and individual differences in clinical samples, which has added another layer of complexity to genetic findings.

DNA methylation is the most widely studied epigenetic mechanism. This DNA modification is found in cytosine residues predominantly in the CpG dinucleotide, and its presence has been correlated with both gene silencing and activation, depending on where it accumulates on the genome (e.g. promoter vs. gene body). Genome-wide DNA methylation changes in peripheral blood have already been associated with psychiatric conditions, including attention-deficit/hyperactivity disorder, schizophrenia, and mood and anxiety disorders, but very few studies have explored them in individuals with substance use disorders. The first studies were conducted in individuals with tobacco ${ }^{2}$ or alcohol substance disorder ${ }^{3}$ but, specifically regarding cocaine, evidence was limited to a single study that explored the state of peripheral DNA methylation in polysubstance abusers. ${ }^{4} \mathrm{~A}$ recent study published in this journal, ${ }^{5}$ however, has identified 186 differentially methylated positions between patients with cocaine use disorder (CUD) and healthy controls using bisulfiteconverted DNA and genome-wide arrays. In this first epigenome-wide association study with CUD patients, the authors used a gene network approach among differentially methylated genes, revealing a pathway affected by CUD and implicated in gene transcription and chromatin regulation. The authors are correct, however, in advising caution in the interpretation of their promising results before they can be replicated in studies with larger samples sizes that include both sexes, especially in association with other relevant clinical variables.

Thus, the contribution of epigenetic studies to the field of neuropsychiatry is exceptionally encouraging. Evidence suggests that at least part of the differentially methylated CpGs detected in the blood of psychiatric patients correlates with postmortem brain methylation levels, strengthening the idea of peripheral DNA methylation as a brain proxy and its use as a useful biomarker. In addition, DNA methylation changes at specific CpGs have also been suggested as powerful biomarkers of aging among patients with mood disorders, which emphasizes the importance of such epigenetic mechanisms in other endophenotypes of relevance for neuropsychiatry.

Our understanding of the complexity of epigenetic research is rapidly increasing with the discovery of new epigenetic markers and the increasing use of deepsequencing techniques and cell-type specific investigations. In this era of "omics", epigenetic investigations have the potential to reveal more and more of the complex molecular signatures of psychiatric disorders. As this happens, special attention should be given to the translation of such discoveries to clinical practice, with the aim of improving patient diagnosis, treatment and prognosis.
Correspondence: Gabriel Rodrigo Fries, Department of Psychiatry and Behavioral Sciences, University of Texas Health Science Center at Houston, 77054 East Rd., Houston, TX, USA.

E-mail: Gabriel.R.Fries@uth.tmc.edu
How to cite this article: Viola TW, Fries GR. A promising era for epigenetic research: revealing the molecular signature of neuropsychiatric disorders. Braz J Psychiatry. 2019;41:469-470. http://dx.doi. org/10.1590/1516-4446-2019-0638 


\section{Disclosure}

The authors report no conflicts of interest.

\section{References}

1 Bredy TW, Wu H, Crego C, Zellhoefer J, Sun YE, Barad M. Histone modifications around individual BDNF gene promoters in prefrontal cortex are associated with extinction of conditioned fear. Learn Mem. 2007;14:268-76.

2 Zeilinger S, Kühnel B, Klopp N, Baurecht H, Kleinschmidt A, Gieger $\mathrm{C}$, et al. Tobacco smoking leads to extensive genome-wide changes in DNA methylation. PLoS One. 2013;8:e63812.
3 Ruggeri B, Nymberg C, Vuoksimaa E, Lourdusamy A, Wong CP, Carvalho FM, et al. Association of protein phosphatase PPM1G with alcohol use disorder and brain activity during behavioral control in a genome-wide methylation analysis. Am J Psychiatry. 2015; 172:543-52.

4 Lax E, Warhaftig G, Ohana D, Maayan R, Delayahu Y, Roska P, et al. A DNA methylation signature of addiction in T cells and its reversal with DHEA intervention. Front Mol Neurosci. 2018:11:322.

5 Camilo C, Maschietto M, Vieira HC, Tahira AC, Gouveia GR, Feio Dos Santos AC, et al. Genome-wide DNA methylation profile in the peripheral blood of cocaine and crack dependents. Braz J Psychiatry. 2019;41:485-93. 